\title{
Universal and Unique Features of Kinesin Motors: Insights from a Comparison of Fungal and Animal Conventional Kinesins
}

\author{
J ochen Kirchner, Günther Woehlke and \\ Manfred Schliwa* \\ Adolf-Butenandt-Institut, Zellbiologie, Universität \\ München, Schillerstr. 42, D-80336 München, Germany \\ ${ }^{*}$ Corresponding author
}

Kinesins are microtubule motors that use the energy derived from the hydrolysis of ATP to move unidirectionally along microtubules. The founding member of this still growing superfamily is conventional kinesin, a dimeric motor that moves processively towards the plus end of microtubules. Within the family of conventional kinesins, two groups can be distinguished to date, one derived from animal species, and one originating from filamentous fungi. So far no conventional kinesin has been reported from plant cells. Fungal and animal conventional kinesins differ in several respects, both in terms of their primary sequence and their physiological properties. Thus all fungal conventional kinesins move at velocities that are $4-5$ times higher than those of animal conventional kinesins, and all of them appear to lack associated light chains. Both groups of motors are characterized by a number of group-specific sequence features which are considered here with respect to their functional importance. Animal and fungal conventional kinesins also share a number of sequence characteristics which point to common principles of motor function. The overall domain organization is remarkably similar. A C-terminal sequence motif common to all kinesins, which constitutes the only region of high homology outside the motor domain, suggests common principles of cargo association in both groups of motors. Consideration of the differences of, and similarities between, fungal and animal kinesins offers novel possibilities for experimentation (e. g., by constructing chimeras) that can be expected to contribute to our understanding of motor function.

Key words: Cargo association / Kinesin / Molecular motors / S equence organization.

\section{Conventional Kinesins: A Brief Summary}

In 1985, a novel type of motor protein was isolated from squid neural tissue (Vale et al., 1985), bovine brain (Brady, 1985) and sea urchin eggs (Scholey et al., 1985) that could move microtubules in vitro in the presence of ATP, and was named kinesin. Since then, kinesins have been found to constitute a superfamily of proteins with now more than 100 members. The defining common denominator of kinesins is the catalytic motor domain, which enables kinesin to hydrolyze ATP in the presence of microtubules. Within members of the superfamily the position of this domain varies: it can be found at either the $\mathrm{N}$ - or $\mathrm{C}$-terminal, or in a central part of the molecule. Within these three subgroups further distinctions can be made, depending on the oligomerization states and homology in the neck regions adjacent to the core motor domains (see Vale and Fletterick, 1997). Using these criteria the kinesin superfamily has been divided into 10 subfamilies.

This structural heterogeneity is thought to reflect functional specifications: kinesins have been found to fulfill various roles in the cell, e. g., as transporters of a wide variety of membranous organelles, chromosomes, and protein complexes (for reviews see Hirokawa, 1998; Lane and Allan, 1998; Steinberg, 1998), and as modulators of the gestalt (structure) of the microtubule cytoskeleton (Sharp et al., 1999) and of mic rotubule stability (Desai et al., 1999). The best studied kinesin subfamily comprises the conventional kinesins. 14 conventional kinesins from 10 different organisms have been sequenced completely, six animal and four fungal species, covering a wide evolutionary spectrum in both kingdoms (Figure 1A, for a review see Hirokawa, 1998). In mouse and man, three different kinesin genes have been found (Nagakawa et al., 1997; Xia et al., 1998). Two of their gene products (KIF5a and KIF5c) are specific for neural tissues, while the third (KIF5b) is expressed ubiquitously. Fungal and animal kinesins form two distinct subgroups within the conventional kinesins. The overall sequence homology is about $30 \%$, while within each subgroup the homology is $45 \%$ (animals) and $60 \%$ (fungi). Conventional kinesins from animals are heterotetramers consisting of two identical motor molecules, the heavy chains (100-130 kDa), and two light chains (60 - $70 \mathrm{kDa})$. Rotary shadow EM images reveal two globular heads, followed by a rod or stalk that is sometimes kinked in the middle, and a fan-shaped tail where the light chains bind in animal kinesins (Hirokawa et al., 1989; 


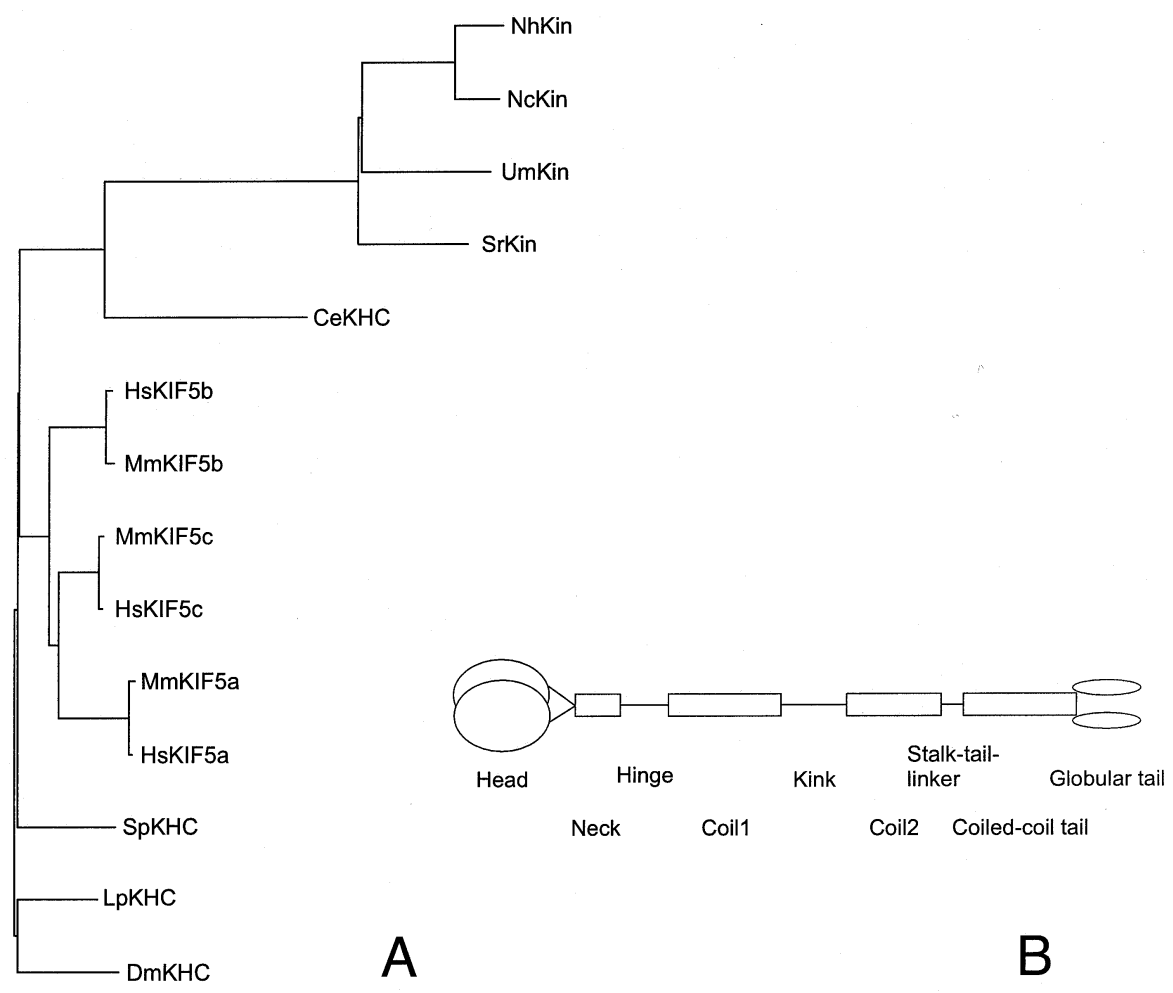

\begin{tabular}{|l|l|l|l|l|l|l|l|l|r|r|}
\hline \multicolumn{10}{|c|}{ Domain homologies of fungal and animal kinesins } \\
\hline Species & Head & Neck & Hinge & Coil1 & Kink & Coil2 & $\begin{array}{l}\text { Stalk-tail- } \\
\text { linker }\end{array}$ & $\begin{array}{l}\text { Coiled-coil } \\
\text { tail }\end{array}$ & $\begin{array}{l}\text { Globular } \\
\text { tail }\end{array}$ & $\begin{array}{l}\text { whole } \\
\text { molecule }\end{array}$ \\
\hline All & $55 \%$ & $35 \%$ & $0 \%$ & $20 \%$ & $10 \%$ & $5 \%$ & $0 \%$ & $60 \%$ & $5 \%$ & $30 \%$ \\
Fungi & $75 \%$ & $75 \%$ & $15 \%$ & $60 \%$ & $35 \%$ & $40 \%$ & $30 \%$ & $80 \%$ & $20 \%$ & $60 \%$ \\
Animals & $70 \%$ & $65 \%$ & $5 \%$ & $45 \%$ & $30 \%$ & $15 \%$ & $30 \%$ & $85 \%$ & $10 \%$ & $45 \%$ \\
Animals* & $75 \%$ & $70 \%$ & $15 \%$ & $60 \%$ & $70 \%$ & $75 \%$ & $70 \%$ & $90 \%$ & $10 \%$ & $65 \%$ \\
\hline
\end{tabular}

${ }^{*}$ Animals without C. elegans

Fig. 1 A Phylogenetic Tree of Conventional Kinesins.

(A) The tree was constructed by the clustal w program of the GCG package. The GenBank accession numbers for the included sequences are: U86521 (NhKin), L47106 (NcKin), U92845 (UmKin), AJ 225894 (SrKin), L19120 (CeKHC), X65873 (HsKIF5b), U86090 (M mKIF5b), AF067180 (MmKIF5C), ABO11103 (HsKIF5C), AF067179 (M mKIF5a), U 06698 (HsKIF5a), X56844 (SpKHC), J 05258 (LpKHC) and M 24441 (DmKHC). (B)A schematic drawing showing the subdomains of conventional kinesins. Non-coiled-coil domains are in the first, coiled-coil domains in the second row. (C) Table showing the homologies of the subdomains of conventional kinesin within the two kingdoms.

Bloom and Endow, 1995). The ability of the kinesin heavy chain $(\mathrm{KHC})$ to form dimers is based on the existence of several stretches that form a coiled-coil according to a prediction based on the Lupas algorithm (Lupas et al., 1991). According to this prediction, the KHC dimer can be divided into 9 subdomains (Figure 1B). These domains are: the catalytic motor domain or head (domain 1 ), neck (domain 2), hinge (domain 3), coil 1 (domain 4), kink (domain 5), coil 2 (domain 6), stalk-tail-linker (domain 7), coiled-coil tail (domain 8) and globular tail (domain 9).

Both the ATP binding site (Kull et al., 1996; Sablin et al., 1996; Gulick et al., 1998; Yang et al., 1990) and the microtubule-binding interface (Woehlke et al., 1997; Alonso et al., 1998) have been mapped to the catalytic motor domain. The neck and its link to the head are important for the determination of the directionality of movement (Henningsen and Schliwa, 1997; Case et al., 1997; Endow and Waligora, 1998). The neck of animal kinesins has been found to form a coiled-coil (Kozielski et al., 1997; Morii et al., 1997; Tripet et al., 1997) and to be involved in kinesin's processivity (Romberg et al., 1998), while both the neck and hinge have been shown to play a role in mechanochemical coupling (Grummt et al., 1998).

The Drosophila conventional kinesin is observed in two conformations resulting in a $9 \mathrm{~S}$ and $6 \mathrm{~S}$ sedimentation constant when placed on sucrose gradients of high and 
low ionic strength, respectively (Hackney et al., 1992). This is now believed to be the consequence of folding at the kink that brings the tail in contact with the head domain, thereby regulating its ATPase activity. C-terminal deletion constructs of KHC coexpressed with different KLC constructs (Verhey et al., 1998) in cultured cells localize to the cell periphery, supporting the model of inactivation by folding of the $\mathrm{C}$-terminus if no cargo is present (Verhey et al., 1998).

From the very beginning, conventional kinesin has been believed to play a role in organelle transport (Vale et al., 1985; Brady, 1985; Schroer et al., 1988). In support of this contention, kinesin has been found to colocalize with a variety of organelles (for a review, see Hirokawa, 1998; Lane and Allan, 1998) including certain types of axonal vesicles (Schnapp et al., 1992), endoplasmic reticulum, Golgi apparatus, mitochondria and lysosomes. Although a potential interaction site of Neurospora crassa kinesin with its cargo has now been located within the coiled-coil tail (Kirchner et al., manuscript submitted), the search for a kinesin receptor has so far proved elusive. The only molecule proposed to be involved in kinesin organelle binding is kinectin (Kumar et al., 1995; Yu et al., 1995), a 160 kDa coiled-coil protein with an $\mathrm{N}$-terminal transmembrane region. It seems to interact with kinesin as well as dynein (Blocker et al., 1997), but its exact role in motor-cargo interaction is still under debate (for reviews see B urkhardt, 1996; Sheetz, 1996). Finally, a kinesin heavy chain knock- out mouse (Tanaka et al., 1998) as well as a Drosophila KHC null mutant (Gho et al., 1992) have been generated. While both of these were lethal, some less severe mutations in the Drosophila KHC gene were viable (Hurd and Saxton, 1996). In contrast, conventional kinesin is nonessential in fungi (Seiler et al., 1997; Wu et al., 1998; Lehmler et al., 1997), and its deletion yields informative phenotypes.

\section{Fungal vs. Animal Conventional Kinesins}

What are the differences and similarities between fungal and animal kinesins, and what can be learned about their function from an analysis of sequence features? Besides the similarities just discussed, there are two functional differences between the groups that stand out. Firstly, fungal kinesins display a much faster in vitro gliding velocity (2.0- $2.5 \mu \mathrm{m}$ per second) than do their animal counterparts $(0.6-0.8 \mu \mathrm{m}$ per second). Secondly, until now, no kinesin light chains have been found to copurify with the heavy chains in fungi. Are these differences (and similarities) reflected in the amino acid sequence? With about $55 \%$ sequence homology, the head is the second-most highly conserved domain of the conventional kinesins (Figure 1C). The head sequences of fungal conventional kinesins comprise groups of amino acids that are highly specific for fungal kinesins and differ significantly
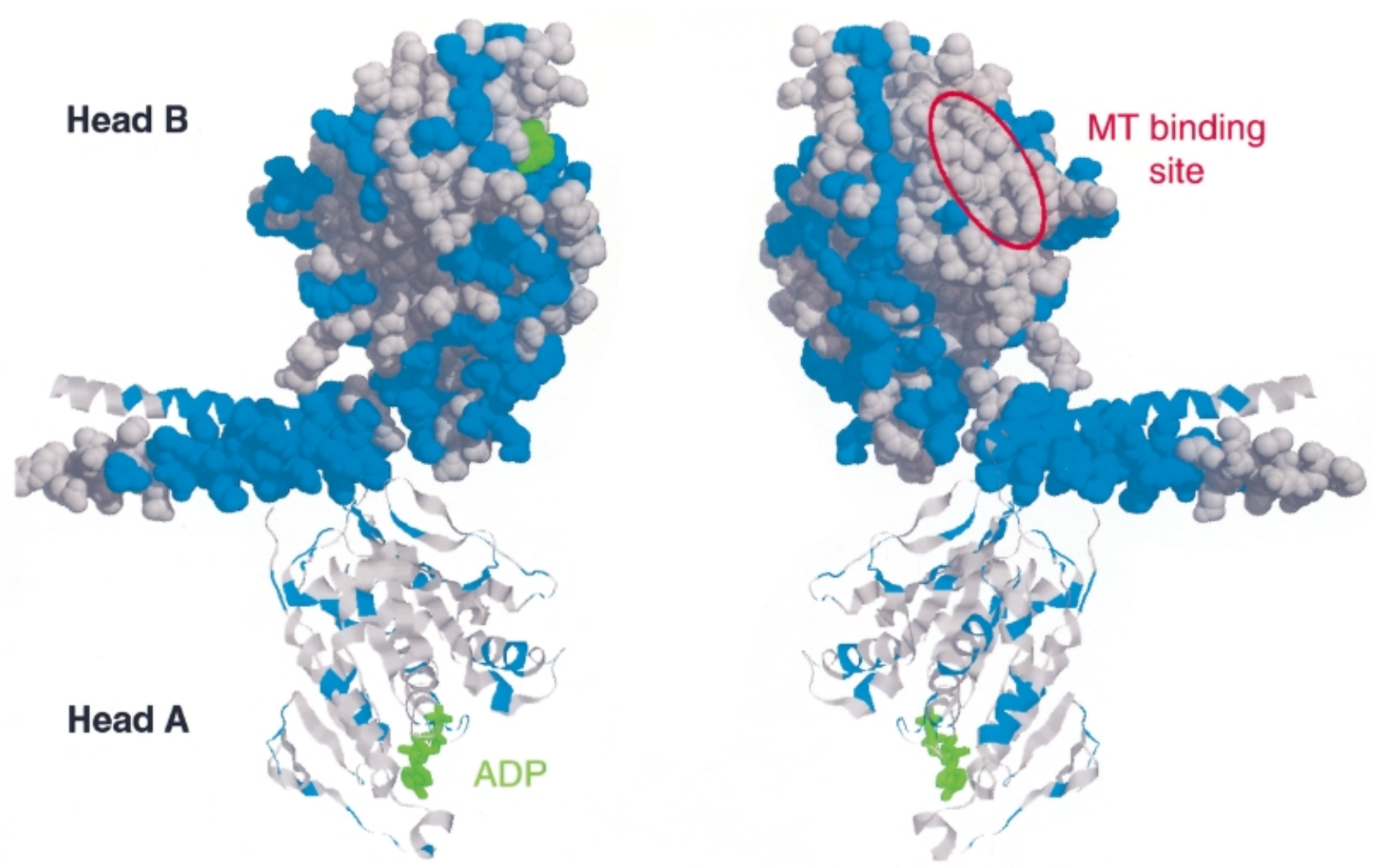

Fig. 2 Crystallographic Model of the Rat Kinesin Motor Domain (Kozielski et al., 1997).

Head $A$ is depicted as a ribbon, head B as a space-filling structure. Positions where fungal kinesins display group-specific sequence features are displayed in blue. The sites of ADP- and microtubule-binding are indicated. 
from their animal relatives. If these specific patches are mapped in the 3D-crystal structure it becomes apparent that all of them are located on the molecule's surface (Figure 2). Whereas catalytically important structures such as P-loop, switch I and II (Vale, 1996) and the L12- $\alpha 5$ microtubule bind ing site are well conserved (as they are for the entire superfamily) the remainder of the molecule displays a patchwork of common and more or less specific residues. Among fungi, parts of helix 1 (amino acid $62-69$ in NcKin), helix 2 (amino acid 121 - 126) and helix 3 (amino acid 185-190) are conserved as well as small groups of residues of the $\beta$-sheets 4 and $5(133-176)$ and loop 10 (217 - 227). The sequence alignments suggest a 7 amino acid insertion before $\beta$-sheet $1 c$. For none of these regions any functional role has been reported so far. The proximity of helix 3 , in particular, to the bound nucleotide may indicate some influence of the fungi-specific exchanges on the enzymatic properties of fungal kinesins, but a simple phylogenetic diversion without functional implications of some of the specific residues cannot be excluded either. Interestingly, the loop 11 which is disordered in all available structures (Kull et al., 1996; Sablin et al., 1996; Sack et al., 1997; Kozielski et al., 1997; Gulick et al., 1998) comprises a subgroup-specific pattern (GKTGASGQT in fun$\mathrm{gi})$. That the $\mathrm{N}$-terminal part of this sequence matches the $\mathrm{P}$-loop sequence motif seems to be a coincidence. It has been suggested that this structure participates in microtubule binding (Sosa et al., 1997) but crucial involvement in communication between the nucleotide and microtubule binding sites is also possible (Vale, 1996; Gulick et al., 1998). Either scenario may imply a functional importance of this motif for the high velocities of fungal kinesins.

One of the most obvious regions of dissimilarity between fungal and animal kinesins is the $\mathrm{N}$-terminal part of the neck. It shows the highest degree of kingdom-specific exchanges within the first two domains (Figure 2). Because it has also been shown to be important for the velocity of movement (Grummt et al., 1998), it is conceivable that the differences observed in this domain are directly linked to this parameter. Additionally, the fungalspecific loop 10 (located between $\beta$-sheets 6 and 7 ; amino acid $217-227$ in NcKin) is found close to the neck region in the crystal structure, suggesting that it is important for head/neck communication and coordination during movement. However, fast gliding velocities may be an additive effect requiring tuning of several structural domains.

The hinge regions show no visible conservation among conventional kinesins, except between very closely related species (e. g., mouse/man homologs), and also vary in length. Within the fungi this region is rich in prolines and glycines, suggesting a high degree of flexibility. Its conformation but not its primary structure seems to be important as the hinge of Drosophila melanogaster can be substituted into SrKin without loss in gliding velocity, whereas a proline-rich region from an unrelated protein cannot (Grummt et al., 1998).

Coil 1 seems to be of relatively uniform length (ca. 130 aa) in all conventional kinesins. Although homology be- tween all kinesins is only about $20 \%$, the homology within the two kingdoms is still quite prominent $(60 \%$ in fungi, $60 \%$ in animals without Caenorhabditis elegans; Figure $1 \mathrm{C}$ ).

In animal conventional kinesins the kink is still well-conserved $(70 \%$ homology for animals without C. elegans) while the kinks of fungal kinesins are about 20 amino acids shorter and only half as conserved ( $35 \%$ homology). Nevertheless, the same back-folding mechanism that is discussed for kinesin from D. melanogaster (Hackney et al., 1992) could also exist in Neurospora crassa, since deletions of the kink results in severely compromised in vivo activity (Kirchner et al., manuscript submitted).

Coil 2 is the most variable region concerning its size. It varies between about 90 residues in $C$. elegans, $N$. crassa and Nectria haematococca, and about 200 residues in animal kinesins. Sequence conservation exists only between close relatives. In animal kinesins, however, the last 30 amino acids are well conserved. This region is strongly suspected to bind the light chains (Diefenbach et al., 1998)

Because of the widely accepted importance of the tail for the association of kinesin with its cargo(es) and the potential regulatory functions localized in this domain, the $3 \mathrm{C}$-terminal domains have been analyzed in more detail (Figure 3). The break in the coiled-coil prediction that marks the transition between the stalk and the tail (stalktail-linker) is still partially conserved in animals, but not in fungi, either in length or in primary structure. An exception are the close relatives $\mathrm{N}$. crassa and $\mathrm{N}$. haematococca. It should be noted that in animals the stalk-tail-linker is located 30-40 amino acids downstream from its fungal counterpart. The region in animal kinesins that aligns with the fungal kinesin stalk-tail-linker contains the light-chain binding site as identified by Diefenbach et al. (1998; highlighted in green in Figure 3). The region in fungal kinesins that aligns with the animal kinesin stalk-tail-linker is highly conserved among fungi and might represent a site of protein protein interaction (highlighted in blue in Figure 3). Whether it represents a site for interaction with light chain equivalents is questionable since no light chains have been found to copurify with $\mathrm{KHC}$ in fungi. Also, this region is already part of the tail coiled-coil of fungal kinesins, only separated from the following conserved region by a discontinuity in the heptad repeat pattern (marked with three blue dashes in Figure 3). The differences in the positioning of this conserved stretch of ca. 30 amino acids in fungi and animals (blue box and green box in Figure 3, respectively) suggests different molecular geometries of the conventional kinesin stalk and tail in fungi and in animals.

One segment of about 50 amino acids in length (red box in Figure 3) clearly stands out in the coiled-coil tail. It is highly conserved between all conventional kinesins and the only large segment outside the catalytic motor domain that exhibits a high degree of homology. Based on an in vivo analysis of mutant kinesins (Kirchner et al., manuscript sub mitted), this region may participate in cargo binding. If confirmed, this would suggest that the basic mech- 

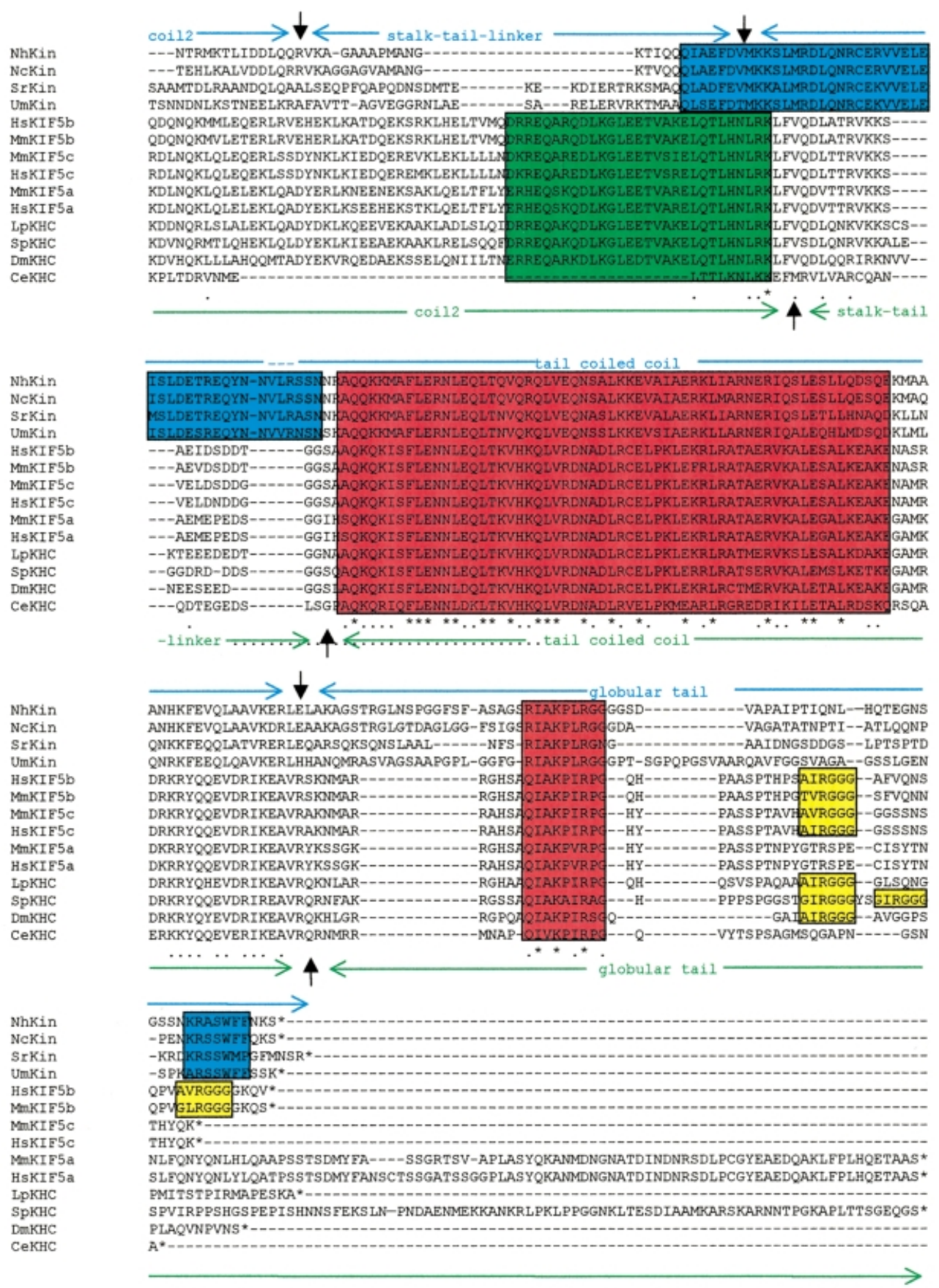

Fig. 3 A Sequence Alignment of the Tail of Conventional Kinesins.

Motifs conserved between all conventional kinesins are highlighted in red. Fungal-specific sequences are highlighted in blue, the animalspecific region thought to represent the light chain binding site is highlighted in green. Domains are marked by blue and green horizontal arrows, respectively, with black vertical arrows indicating the boundaries. An irregularity in the fungal tail coiled-coil is marked with three blue dashes. A motif occuring only in some animal kinesins is shown in yellow.

anisms of cargo association are conserved in both animal and fungal kinesins.

The globular tail domain is of variable length. Almost nonexistent in C. elegans, it extends over ca. 120 amino acids in KIF 5 a and sea urchin kinesin, and about 70 amino acids in fungal kinesins (see Figure 3 ). It contains a short motif conserved in all conventional kinesins (IAKP$(\mathrm{L} / \mathrm{I} / \mathrm{V}) \mathrm{R} \times \mathrm{G}$, highlighted in red in Figure 3 ) as well as a protein kinase A consensus site $\underline{K} R S \underline{S W}$ ) conserved only in fungal kinesins (boxed in blue in Figure 3). Another inter- esting feature is the motif $(A / T / G)(V / I / L) R G G G$, which occurs even twice in KIF5b and SpKHC (highlighted in yellow in Figure 3). Both the common and class-specific conserved motifs of conventional kinesins might be associated with different aspects of kinesin regulation. The $(A / T / G)(I / V / L) R G G G$ motif is reminiscent of the RGG repeat that constitutes an RNA binding motif (Burd and Dreyfuss 1994). However, the RGG repeat usually occurs 5 times in RNA-binding proteins, so the question of an RNA-binding site in conventional kinesins remains open. 


\section{Comparison of Kinesin Mutants from Animals and Fungi}

While all animal kinesin knock-outs that have been performed so far (C. elegans, D. melanogaster, mouse) were lethal because of damage to neural tissues (Gho et al., 1992; Saxton et al., 1991; Patel et al., 1993; Tanaka et al., 1998) all three fungal kinesin null mutants from N. crassa (Seiler et al., 1997), N. haematococca (Wu et al., 1998) and Ustilago maydis (Lehmler et al., 1997) are viable and display informative phenotypes. In N. crassa the most prominent feature of the kinesin null mutant is its reduced hyphal growth speed (1.4 vs. $6.8 \mathrm{~cm} /$ day, Seiler et al., 1997). Also, the hyphae become thicker and less regular in shape, and the Spitzenkörper, an accumulation of vesicles at the growing tip, is much reduced. However, the transport of microscopically visible organelles in hyphae appears normal (Seiler et al., 1997). In the N. haematococca kinesin null mutant the growth rate is reduced to $50 \%$ and the shape of hyphae also becomes more irregular, but hyphae appear thinner and the distribution of mitochondria near the growing tip seems to be disturbed (Wu et al., 1998). The Spitzenkörper is smaller and loses its central positioning in the growing tip. In U. maydis, the dikaryon of the null mutant exhibits delayed growth after mating, and plant pathogenicity is almost completely abolished (Lehmler et al., 1997). Also, the organization of the subapical part of the growing tip is altered.

In animal kinesin null mutants two interesting cellular defects have been observed. In D. melanogaster, the kinesin null mutant shows axonal swellings packed with supposedly stalled organelles (Hurd and Saxton, 1996), while in mouse KIF $5 \mathrm{~b}$ null mutants cells cultured from the visceral yolk sack displayed a perinuclear clustering of mitochondria (Tanaka et al., 1998).

The common denominator of all these null mutants is defective coordination of a process presumably involving the transport of membranous organelles. Interestingly, in two cases conventional kinesin has been found to play a role in mitochondrial distribution (Wu et al., 1998; Tanaka et al., 1998), while in another study the transport of organelles visible in the light microscope was unaffected (Seiler et al., 1997). Thus, so far no unifying picture has emerged, and the participation of kinesin in a variety of cellular processes may be fine-tuned in a class-specific and possibly even species-specific fashion.

\section{Conclusions}

The comparison of animal and fungal conventional kinesins allows to differentiate between basic features of kinesin function (conserved between all conventional kinesins) and more specialized aspects that have evolved in a subset of conventional kinesins to meet specialized needs. The catalytic and microtubule-binding activities, the subdomain pattern (Figure 1B), the folding mechanism to inhibit the ATP ase, and the prospective cargo binding site all seem to be expressed in a similar fashion in animal and fungal kinesins.

On the other hand, fungal kinesins apparently lack light chains, which could reflect the fact that, as primitive multicellular organisms in which basically a single cell type is reiterated, they can do with a less complex regulatory machinery for kinesin. The higher speed of fungal kinesins could be necessary to maintain the extremely fast longitudinal growth of N. crassa (ca. $1 \mu \mathrm{m} / \mathrm{s}$ under optimal conditions).

Finally, one important question stands out. Conventional kinesins have been found to be associated with, or affect the distribution of, a variety of organelles in different organisms with no unifying pattern emerging. While conventional kinesin knock-outs affect similar organelles in highly divergent species (e. g., mitochondria in the mouse and $\mathrm{N}$. haematococca), even in closely related species the effects may vary (e. g., N. haematococca and N. crassa). So far the available evidence neither suggests a clear difference between animals and fungi, nor a striking similarity between closely related species. On the other hand, the region in the tail domain conserved between all conventional kinesins seems to be important for cargo binding and can be expected to associate with a similarly conserved partner on the cargo. To resolve this apparent contradiction will be an exciting task in the future.

\section{References}

Alonso, M.C., van Damme, J ., Vandekerckhove, J ., and Cross, R.A. (1998). Proteolytic mapping of kinesin/ncd-microtubule interface: nucleotide-dependent conformational changes in the loops L8 and L12. EMBO J . 17, 945 - 951.

Blocker, A., Severin, F.F., Burkhardt, J .K., Bingham, J .B., Yu, H., Olivo, J .C., Schroer, T.A., Hyman, A.A., and Griffiths, G. (1997). Molecular requirements for bi-directional movement of phagosomes along microtubules. J. Cell Biol. 137, 113- 129.

Bloom, G.S., and Endow, S.A. (1995). Motor proteins 1: Kinesins. Protein profile 1, 1059-1116.

Brady, S.T. (1985). A novel brain ATPase with properties expected for the fast axonal transport motor. Nature 317, 73- 75.

Burd, C.G., and Dreyfuss, G. (1994). Conserved structures and diversity of functions of RNA-binding proteins. Science 265 , $615-621$.

Burkhardt, J . (1996). In search of membrane receptors for microtubule-based motors - is kinectin a kinesin receptor? Trends Cell Biol. 6, 127-131.

Case, R.B., Pierce, D.W., Hom-Boher, N., Hart, C.L., and Vale, R.D. (1997). The directional preference of kinesin motors is specified by an element outside the catalytic motor domain. Cell 90, 1-20.

Desai, A., Verma, S., Mitchison, T.J ., and Walczak, C.E. (1999). Kin I kinesins are microtubule-destabilizing enzymes. Cell 96, $69-78$.

Diefenbach, R.J ., Mackay, J .P., Armati, P.J ., and Cunningham, A.L. (1998). The C-terminal region of the stalk domain of ubiquitous human kinesin heavy chain contains the binding site for kinesin light chain. Biochemistry 37, 16663-16670.

Endow, S.A., and Waligora, K.W. (1998). Determinants of kinesin motor polarity. Science 281, 1200 - 1202. 
Gho, M., McDonald, K., Ganetzky, B., and Saxton, W.M. (1992). Effects of kinesin mutations on neuronal functions. Science 258, 313-316.

Grummt, M., Woehlke, G., Henningsen, U., Fuchs, S., Schleicher, M., and Schliwa, M. (1998). Importance of a flexible hinge near the motor domain in kinesin-driven motility. EMBO J . 17, $5536-5542$.

Gulick, A.M., Song, H., Endow, S.A., and Rayment, I. (1998). X-ray crystal structure of the yeast Kar 3 motor domain complexed with M gATP to 2.3 \& resolution. Biochemistry 37, 1769- 1776.

Hackney, D.D., Levitt, J .D., and Suhan, J . (1992). Kinesin undergoes a $9 \mathrm{~S}$ to $6 \mathrm{~S}$ conformational transition. J. Biol. Chem. 267, $8696-8701$.

Henningsen, U., and Schliwa, M. (1997). Reversal in the direction of a movement in a molecular motor. Nature 389, 93 - 96.

Hirokawa, N., Pfister, K.K., Yorifuji, H., Wagner, M.C., Brady, S.T., and Bloom, G.S. (1989). Submoleculardomains of bovine brain kinesin identified by electron microscopy and monoclonal antibody decoration. Cell 56, 867-878.

Hirokawa, N. (1998). Kinesin and dynein superfamily proteins and the mechanism of organelle transport. Science 279, 519- 526.

Hurd, D.D., and Saxton, W.M. (1996). Kinesin mutations cause motor neuron disease phenotypes by disrupting fast axonal transport in Drosophila. Genetics 144, 1075- 1085.

Kozielski, F., Sack, S., Marx, A., Thormählen, M., Schönbrunn, E., Biou, V., Thompson, A., Mandelkow, E.M., and Mandelkow, E. (1997). The crystal structure of dimeric kinesin and implications for mic rotubule-dependent motility. Cell 91, 985 - 994.

Kull, F.J ., Sablin, E.P., Lau, R., Fletterick, R.J., and Vale, R.D. (1996). Crystal structure of the kinesin motor domain reveals a structural similarity to myosin. Nature 380, 550 - 555.

Kumar, J., Yu, H., and Sheetz, M.P. (1995). Kinectin, an essential anchor for kinesin-driven vesicle motility. Science 267, 1834 1837.

Lane, J., and Allan, V. (1998). Microtubule-based membrane movement. Biochimica et Biophysica Acta 1376, 27 - 55.

Lehmler, C., Steinberg, G., Snetselaar, K.M., Schliwa, M., Kahmann, R., and Bölker, M. (1997). Identification of a motor protein required for filamentous growth in U. maydis. EMBO J . 16, $3464-3473$.

Lupas, A., Van Dyke, M., and Stock, J . (1991). Predicting coiledcoils from protein sequences. Science 252, $1162-1164$.

Morii, H., Takenawa, T., Arisaka, F., and Shimizu, T. (1997). Identification of kinesin neck region as a stable $\alpha$-helical coiledcoil and its thermodynamic characterization. Biochemistry 36 , $1933-1942$.

Nagakawa, T., Tanaka, Y., Matsuoka, E., Kondo, S., Okada, Y., Noda, Y., Kanai, Y., and Hirokawa, N. (1997). Identification and classification of 16 new kinesin superfamily (KIF) proteins in mouse genome. Proc. Natl. Acad. Sci. USA 94, 9654 - 9659.

Patel, N., Thierry-Mieg, D., and Mancillas, J .R. (1993). Cloning by insertional mutagenesis of a cDNA encoding $C$. elegans kinesin heavy chain. Proc. Natl. Acad. Sci. USA 90, 9181 - 9185.

Romberg, L., Pierce, D.W., and Vale, R.D. (1998). Role of the kinesin neck region in processive microtubule-based motility. J. Cell Biol. 140, $1407-1416$.

Sablin, E.P., Kull, F.J ., Cooke, R., Vale, R.D., and Fletterick, R.J . (1996). Crystal structure of the motor domain of the kinesinrelated motor ncd. Nature 380, 555- 559.

Sack, S., Müller, J ., Marx, A., Thormählen, M., Mandelkow, E.M., Brady, S.T., and Mandelkow, E. (1997). X-ray structure of motor and neck domains from rat brain kinesin. Biochemistry 36 , $16155-16165$.

Saxton, W.M., Hicks, J ., Goldstein, L.S.B., and Raff, E.C. (1991). Kinesin heavy chain is essential for viability and neuromuscular functions in Drosophila, but mutants show no defect in mitosis. Cell 64, 1093-1102.

Schnapp, B.J ., Reese, T.S., and Bechtold, R. (1992). Kinesin is bound with high affinity to squid axon organelles that move to the plus-end of microtubules. J. Cell Biol. 119, 389- 399.

Scholey, J.M., Porter, M.E., Grissom, P.M., and Mclntosh, J .R. (1985). Identification of kinesin in sea urchin eggs, and evidence for its localization in the mitotic spindle. Nature 318 , 483-486.

Schroer, T.A., Schnapp, B.J., Reese, T.S., and Sheetz, M.P. (1988). The role of kinesin and other soluble factors in organelle movement along microtubules. J. Cell Biol. 107, 1785 - 1792.

Seiler, S., Nargang, F., Steinberg, G., and Schliwa, M. (1997). Kinesin is essential for cell morphogenesis and polarized secretion in N. crassa. EMBO J . 16, 3025 - 3034.

Sharp, D.J ., McDonald, K.L., Brown, H.M., Matthies, H.J ., Walczak, C., Vale, R.D., Mitchison, T.J ., and Scholey, J .M. (1999). The bipolar kinesin, KLP61F, cross-links microtubules within interpolar bundles of Drosophila embryonic mitotic spindles. J. Cell Biol. 144, $125-138$.

Sheetz, M.P. (1996). Microtubule motor complexes moving membranous organelles. Cell Struc. Funct. 21, 369- 373.

Sosa, H., Dias, D.P., Hoenger, A., Whittaker, M., Wilson-Kubalek, E., Sablin, E., Fletterick, R.J., Vale, R.D., and Milligan, R.A. (1997). A model for the microtubule-Ncd motor protein complex obtained by cryo-electron microscopy and image analysis. Cell 90, 217- 224.

Steinberg, G. (1998). Organelle transport and molecular motors in fungi. Fungal Genet. Biol. 24, $161-177$.

Tanaka, Y., Kanai, Y., Okada, Y., Nonaka, S., Takeda, S., Harada, A., and Hirokawa, N. (1998). Targeted disruption of mouse conventional kinesin heavy chain, kif5b, results in abnormal perinuclear clustering of mitochondria. Cell 93, 1147- 1158.

Tripet, B., Vale, R.D., and Hodges, R.S. (1997). Demonstration of coiled-coil interactions within the kinesin neck region using synthetic peptides. J. Biol. Chem. 272, 8946-8956.

Vale, R.D. (1996). Switches, latches and amplifiers: common themes of $\mathrm{G}$ proteins and molecular motors. J . Cell Biol. 135, $291-302$.

Vale, R.D., and Fletterick, R. (1997). The design plan of kinesin motors. Annu. Rev. Cell Dev. Biol. 13, 745 - 777.

Vale, R.D., Reese, T.S., and Sheetz, M.P. (1985). Identification of a novel force-generating protein, kinesin, involved in microtubule based motility. Cell 42, 39-50.

Verhey, K.J ., Lizotte, D.L., Abramson, T., Barenboim, L., Schnapp, B.J ., and Rapoport, T.A. (1998). Light chain-dependent regulation of kinesin's interaction with microtubules. J. Cell Biol. 143, $1053-1066$

Woehlke, G., Ruby, A.K., Hart, C.L., Ly, B., Hom-Booher, N., and Vale, R.D. (1997). Microtubule interaction site of the kinesin motor. Cell 90, $207-216$.

Wu, Q., Sandrock, T.M., Turgeon, B.G., Yoder, O.C., Wirsel, S.G., and Aist, J.R. (1998). A fungal kinesin required for organelle motility, hyphal growth and morphogenesis. Mol. Biol. Cell 9, $89-101$.

Xia, C., Rahman, A., Yang, Z., and Goldstein, L.S.B. (1998). Chromosomal localization reveals three kinesin heavy chain genes in mouse. Genomics 52, 209- 213.

Yang, J .T., Saxton, W.M., Stewart, R.J ., Raff, E.C., and Goldstein, L.S.B. (1990). Evidence that the head of kinesin is sufficient for force generation and motility in vitro. Science 249, $42-47$.

Yu, H., Nicchitta, C.V., Kumar, J ., Becker, M., Toyoshima, I., and Sheetz, M.P. (1995). Characterization of kinectin, a kinesinbinding protein: primary sequence and $\mathrm{N}$-terminal topogenic signal analysis. Mol. Biol. Cell 6, 171- 183. 